\title{
Pain management and prevention of suicide in the COVID-19 era
}

\author{
Soroush Pakniyat-Jahromi ${ }^{1}$ [ $\cdot$ Leo Sher $^{2,3}$
}

Received: 11 October 2020 / Accepted: 24 February 2021 / Published online: 5 March 2021

(c) Springer-Verlag GmbH, DE part of Springer Nature 2021

Keywords Chronic pain $\cdot$ COVID-19 $\cdot$ Pandemic era $\cdot$ Suicide

Pain is a frequent symptom in hospitalised patients and outpatients. Chronic pain is reported to be the most common complaint in primary care setting, affecting more than 100 million people in the USA with an annual cost greater than $\$ 635$ billion [1]. Many patients are suffering from pain because of different illnesses including but not limited to cancer, musculoskeletal pain conditions (i.e., fibromyalgia, back pain), and migraine. Social isolation and other COVID19-related issues may decrease the quality of life in patients with chronic pain [2]. A great proportion of chronic pain management services (CPMS) are generally considered non-urgent in this era, and hospital resources and professionals employed in CPMS are used to treat patients in the COVID-19 units and emergency rooms [3]. Clinics are less accessible and waiting times are prolonged.

Pain management is usually complicated and difficult as there is no one size fit for all and every patient should be managed differently. Many patients are not being sufficiently treated because of different reasons. Some are not fully evaluated and are misdiagnosed; some are not on the right medications or are undertreated due to adverse reactions to medications or the policy of shifting away from opioids in general due to the reports of overdoses with prescribed opioids. The USA Centers for Disease Control and Prevention (CDC) reported that about 42,000 opioid overdoses resulted in death including an unknown number of suicides in 2016 [3]. The issue of identifying the cause of death may result in underreporting suicides due to opioid overdose. In 2016, the

Soroush Pakniyat-Jahromi

sjahromi@bronxcare.org; soroushpakniyat@gmail.com

1 BronxCare Health System, 1276 Fulton Avenue, Bronx, NY 10456, USA

2 James J. Peters Veterans' Administration Medical Center, Bronx, NY, USA

3 Icahn School of Medicine at Mount Sinai, New York, NY, USA
CDC reported 44,965 suicides in the United States and 15\% were carried out by drug overdose [4]. There is an overlap in the neural system of physical and psycho-social pain and they are both linked with suicidal behaviors. In a study in the UK, persons with suicidal ideation (SI) or history of suicide attempt (SA) used more opioids than affective individuals without SI/SA. Both groups used opioids mainly to relieve from psycho-social pain, rather than physical pain [5]. A study in Italy showed that in chronic pain patients, higher aggression and impulsivity might increase the risk of suicide [6]. It is worth mentioning that opioids are also cough suppressants and their use during COVID-19 era should be limited as they could mask the symptoms of COVID-19 infection and delay diagnosis and result in more spread of the disease.

Considerable evidence suggests that pain is an independent contributor to suicidal behaviors [7, 8]. A study in 18 USA states from 2003 to 2014 reported that nine percent of suicide victims had a history of chronic pain, according to their medical records [8]. Many studies have focused on the type, duration, location, and severity of pain in suicide cases; however, in one study, these factors were not directly related to suicidal behavior but psycho-social factors related to physical pain such as pain catastrophising, helplessness, and mental defeat were associated with suicidal behavior in pain patients [9]. Elman et al. [10] have suggested that pain can result in alteration in brain circuits leading to an imbalance of the reward (depressed function) and anti-reward (sensitised function) networks in the brain. They recommended that any intervention that would help restore this balance in chronic pain patients, such as adequate pain management with non-opioid medications, could decrease suicide risk.

Due to the association of pain with suicidal ideation and attempts, comprehensive suicide screening and assessment of patients with chronic pain is a necessity and individuals with elevated suicide risk should be referred to psychiatrists for a more comprehensive assessment and treatment. 
Education is a key factor, not only for clinicians and healthcare personnel, but also for the public to be aware of the important relation of pain with suicide. Families and on a bigger scale, communities, can be engaged in preventing suicide by referring individuals suffering from pain to the hospital for further evaluation and suicide prevention interventions. Poor management of chronic pain during the COVID-19 era could lead to hopelessness, social detachment, unemployment, insomnia, poor quality of life and depression, resulting in self-medicating behaviors and in some cases leading to suicidal behaviors. During pandemics such as COVID-19, CPMS should remain available. Telemedicine can be used effectively for pain evaluation and management. Practitioners at every level should perform suicide risk assessment during pain evaluation and triage, either in person, or by telemedicine. The practitioners at pain management clinics and psychiatrists should work together and individuals with elevated suicide risk should be referred to psychiatrists for further evaluation and continuation of care. Suicide prevention is a task for all clinicians, not just mental health professionals. More research is warranted to develop better screening tools for pain and safer treatments for individuals suffering from pain and to identify patients at a greater risk for suicide.

Funding No funding or sponsorship was received for this study.

Data availability Not applicable.

Code availability Not applicable.

\section{Declarations}

Conflict of interest The authors have no conflicts of interest to declare.

\section{References}

1. Institute of Medicine (2011) Relieving pain in America a blueprint for transforming prevention, care, education, and research. The National Academies Press, Washington, D.C. https://doi. org/10.17226/13172

2. Kersebaum D, Fabig SC, Sendel M, Sachau J, Lassen J, Rehm S, Hüllemann P, Baron R, Gierthmühlen J (2020) The early influence of COVID-19 pandemic-associated restrictions on pain, mood, and everyday life of patients with painful polyneuropathy. J Pain Rep 5(6):e858. https://doi.org/10.1097/PR9.0000000000000858

3. Piraccini E, Byrne H, Taddei S (2020) Chronic pain management in COVID-19 era. J Clin Anesth 65:109852. https://doi. org/10.1016/j.jclinane.2020.109852

4. Oquendo MA, Volkow ND (2018) Suicide: a silent contributor to opioid-overdose deaths. N Engl J Med 378:1567-1569. https:// doi.org/10.1056/NEJMp1801417

5. Calati R, Olié E, Ritchie K, Artero S, Courtet P (2017) Suicidal ideation and suicide attempts in the elderly associated with opioid use and pain sensitivity. Psychother Psychosom 86:373-375. https ://doi.org/10.1159/000478021

6. Margari F, Lorusso M, Matera E, Pastore A, Zagaria G, Bruno F, Puntillo F, Margari L (2014) Aggression, impulsivity, and suicide risk in benign chronic pain patients - a cross-sectional study. Neuropsychiatr Dis Treat 10:1613-1620. https://doi.org/10.2147/ NDT.S66209

7. Calati R, Bakhiyi CL, Artero S, Ilgen M, Courtet P (2015) The impact of physical pain on suicidal thoughts and behaviors: metaanalyses. J Psychiatr Res 71:16-32. https://doi.org/10.1016/j.jpsyc hires.2015.09.004

8. Petrosky E, Harpaz R, Fowler K, Bohm MK, Helmick CG, Yuan K, Betz CJ (2018) Chronic pain among suicide decedents, 2003 to 2014: findings from the national violent death reporting system. Ann Intern Med 169:448-455. https://doi.org/10.7326/M18-0830

9. Racine M (2018) Chronic pain and suicide risk: a comprehensive review. Prog Neuropsychopharmacol Biol Psychiatry 87:269-280. https://doi.org/10.1016/j.pnpbp.2017.08.020

10. Elman E, Borsook D, Volkow ND (2013) Pain and suicidality: insights from reward and addiction neuroscience. J Prog neurobiol 109:1-27. https://doi.org/10.1016/j.pneurobio.2013.06.003 